\title{
Computation and visualization of the MacAdam limits for any lightness, hue angle, and light source
}

\author{
Francisco Martínez-Verdú, Esther Perales, Elisabet Chorro, Dolores de Fez, and Valentín Viqueira \\ Department of Optics, University of Alicante, Carretera de San Vicente del Raspeig s/n 03690, \\ Alicante, Spain \\ Eduardo Gilabert \\ Department of Paper and Textile Engineering, Technical University of Valencia, Plaza de Ferrándiz y Carbonell, \\ s/n 03801 Alcoy, Spain
}

Received March 14, 2006; revised November 23, 2006; accepted December 7, 2006; posted January 3, 2007 (Doc. ID 68782); published May 9, 2007

\begin{abstract}
We present a systematic algorithm capable of searching for optimal colors for any lightness $L^{*}$ (between 0 and 100), any illuminant (D65, F2, F7, F11, etc.), and any light source reported by CIE. Color solids are graphed in some color spaces (CIELAB, SVF, DIN99d, and CIECAM02) by horizontal (constant lightness) and transversal (constant hue angle) sections. Color solids plotted in DIN99d and CIECAM02 color spaces look more spherical or homogeneous than the ones plotted in CIELAB and SVF color spaces. Depending on the spectrum of the light source or illuminant, the shape of its color solid and its content (variety of distinguishable colors, with or without color correspondence) change drastically, particularly with sources whose spectrum is discontinuous and/or very peaked, with correlated color temperature lower than $5500 \mathrm{~K}$. This could be used to propose an absolute colorimetric quality index for light sources comparing the volumes of their gamuts, in a uniform color space. (C) 2007 Optical Society of America

OCIS codes: $330.1730,330.4060,330.5020,120.5240,230.6080,300.6170$.
\end{abstract}

\section{INTRODUCTION}

The human perception of color is essentially trivariant, so all perceptible and distinguishable colors are threedimensionally distributed, and they shape a volumetric form that is named the color solid. ${ }^{1-3}$ In the upper and lower vertices are the absolute or perceptual white and black, respectively. The colors shaping the intermediate frontiers, obviously with the maximum colorfulness, are named optimal colors, and these were exhaustively studied by MacAdam ${ }^{4,5}$ in 1935. He followed the work by Schrödinger ${ }^{6}$ in 1920 and Rösch ${ }^{7}$ in 1929, who developed the preliminary theory of the optimal colors. For this reason, the Rösch-MacAdam color solid borders are also known as MacAdam limits. MacAdam calculated the optimal chromaticity loci for several luminance factors of the CIE-1931 XYZ standard observer for the A, C, and D65 illuminants. The color solid can be calculated in any color space, although MacAdam worked in the CIE $x y$ chromaticity diagram. ${ }^{8-10}$ Since the CIE- $X Y Z$ color space is not visually uniform, it is better to calculate the color solid in more perceptually uniform color spaces. A uniform color space, widely used in industry, is the CIE$L^{*} a^{*} b^{*}$, which allows one to visualize the color solid in a more realistic way. At present, newer and more perceptually uniform color spaces, such as $\mathrm{SVF}^{11}{ }^{11} \mathrm{DIN} 99 \mathrm{~d},{ }^{12}$ and CIECAM02, ${ }^{13}$ are available, so we are going to plot the color solid in these color spaces and analyze it with profiles of constant lightness and hue angle for several illuminants and light sources.
As was said above, the optimal colors have maximum colorfulness for a given luminance factor. The initial studies of Schrödinger ${ }^{6}$ and Rösch ${ }^{7}$ were summarized by MacAdam in the following theorem: the maximum attainable purity for a material, from a specific given visual efficiency and wavelength, can be obtained if the spectrophotometric curve has as possible values zero or one only, with solely two transitions between these two values in all the visible spectrum. In 1935 MacAdam demonstrated this theorem ${ }^{4}$ by assuming an equivalence between this problem and the calculation of the gravity center in additive color mixing. Therefore, the Rösch-MacAdam color solid can be understood as the color space derived from the color-matching functions. ${ }^{8}$

Two types of optimal colors are possible: type 1, with mountainlike spectral profiles, and type 2 , with valleylike spectral profiles. Figure 1 shows several examples of both types of color stimuli, all of them with the same luminance factor under the equienergetic illuminant encoded by the CIE-1931 XYZ standard observer. The optimal colors do not really exist; that is, they are not found in nature and cannot be obtained by means of colorant formulation. Nevertheless, they serve to delimit the color solid of the human perception and to evaluate the colorimetric quality of colorants ${ }^{8-10}$ : when colorants are near the MacAdam limits, a greater range of reproducible colors (color gamut) can be obtained. For instance, Pointer ${ }^{14,15}$ used in 1980 these colorimetric data for comparing several industrial color gamuts. 


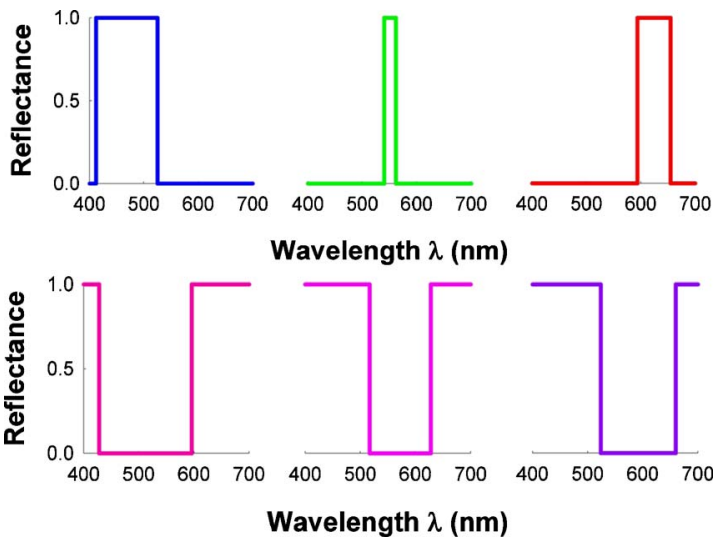

Fig. 1. (Color online) Six examples of optimal colors (left: type 1; right: type 2) with luminance factor $Y=20 \%$ under illuminant $\mathrm{E}$ and the CIE $1931 X Y Z$ standard observer. The transition wavelengths $\lambda_{1}$ and $\lambda_{2}$ are, from left to right, as follows: 412.1-525.2, $540.0-562.0, \quad 594.0-654.7, \quad 428.0-596.0, \quad 517.1-628.0$, and $524.0-660.1 \mathrm{~nm}$.

The original MacAdam's algorithm, based on the calculation of the colorimetric purity, does not search systematically all the optimal colors of the visible spectrum for a specific luminance factor. This means that the MacAdam limits plotted in the current literature are interpolated curves from a discrete and reduced set of original data. Moreover, the most usual illuminants in the literature $^{8,9,16}$ are always A, C, D65, and E, with $Y$ values above $10 \%$.

We present in this work what we believe to be a new algorithm for systematically searching optimal colors for any illuminant (type F, P, D, etc.) and even for real lamps (discharge, fluorescents, LED, etc.), independently of the luminance factor $Y$ (lightness $L^{*}$ ) in the ]0, 100[ range. In this way, the color solid should be completely graphed in several new color spaces, and we can determine how its shape and its content, associated with the variety of distinguishable color sensations, with or without perceptual correspondence, depend on the illuminant-light source.

\section{METHODS}

Our algorithm is composed of two subalgorithms: one for calculating type 1 optimal colors and the other for calcu- lating type 2 optimal colors. By default, the algorithm uses the following data:

(a) The visible spectrum range, for instance, from 380 to $780 \mathrm{~nm}$.

(b) The spectral sampling, $N$, in this case is equal to $0.1 \mathrm{~nm}$.

(c) The color-matching functions of the CIE-1931 XYZ standard observer ${ }^{17}$ (with $\Delta \lambda=1 \mathrm{~nm}$ ), adequately interpolated to fit the spectral sampling (with $\Delta \lambda=0.1 \mathrm{~nm}$ ). Using typical algebraic notation in colorimetry, the CIE color-matching functions are described by $\mathbf{T}$ $=\left[\begin{array}{lll}\overline{\mathbf{x}} & \overline{\mathbf{y}} & \overline{\mathbf{z}}\end{array}\right]_{4001 \times 3}$. Since these curves are smooth, linear interpolation is sufficient. ${ }^{18}$

(d) The spectral power distribution or spectrum $S(\lambda)$ of the illuminant, sampled at the same rate as the colormatching functions. In this way, the weighting tables for any illuminant and CIE-1931 observer combination are computed by $\mathbf{T}^{\prime}=\mathbf{T} \cdot \operatorname{diag}(\mathbf{S})$, where $\operatorname{diag}(\mathbf{S})$ is the diagonal matrix of the illuminant vector $\mathbf{S}$. In this case, we have used spline instead of linear interpolation, following the recommendations of the $\mathrm{CIE},{ }^{18}$ because the spectral curves for some illuminants and light sources curves are not so smooth.

(e) The lightness value $L^{*}$ with tolerance $\Delta L^{*}$. We use these data instead of the luminance factor $Y$ because we want to analyze the color solid at constant lightness planes. The tolerance value $\Delta L^{*}$ guarantees that optimal colors obtained for a certain lightness value are distributed in a constant lightness plane, whose thickness (that is, the lightness difference between the lightest and the darkest optimal colors) does not surpass $\Delta L^{*}$. To work in this way, the dependence of $L^{*}$ on $Y$ for all the range of the luminance factor must be taken into account:

$$
L^{*}=\left\{\begin{array}{ll}
903.3 \frac{Y}{Y_{n}}, & \text { if } Y / Y_{n} \leqslant 0.008856 \\
116\left(\frac{Y}{Y_{n}}\right)^{1 / 3}-16, & \text { if } Y / Y_{n}>0.008856
\end{array},\right.
$$

with $Y_{n}=100$ for the illuminant; if $\Delta L^{*}=f(Y \pm \Delta Y)-f(Y)$ is constant, then

$$
\Delta Y=\left\{\begin{array}{ll}
\frac{100}{903.3} \Delta L^{*}, & \text { if } Y(\%) \leqslant 0.8856 \\
100\left[\left(\frac{\Delta L^{*}}{116}+\left(\frac{Y}{100}\right)^{1 / 3}\right)^{3}-\frac{Y}{100}\right], & \text { if } Y(\%)>0.8856
\end{array} .\right.
$$

With these preliminaries, for each fixed lightness values $L^{*}$ under any illuminant, our routine systematically finds the wavelengths $\lambda_{1}$ and $\lambda_{2}$, where the sudden change of reflectance or transmittance happens (from 0 to 1 , or opposite). That is, the spectra of the optimal colors in
Fig. 1 differ in the center and width but not height (always 0 or 1 ).

This algorithm systematically searches the optimal colors along the selected spectral range. If the chosen spectral range is, for example, from 380 to $780 \mathrm{~nm}$ in steps of 
$0.1 \mathrm{~nm}$, the algorithm has to look for all the possible pairs $\lambda_{1}-\lambda_{2}$ within $8,002,000$ colorimetrically possible combinations. Obviously, this algorithm, for each lightness value $L^{*}$ and optimal color type, has a considerable computational cost. For example, with the algorithm implemented in MATLAB, the average computing time for each optimal color type and lightness is approximately $1 \mathrm{~h}$ with a Pentium IV computer. Obviously, this mainly depends on the wavelength step $\Delta \lambda$ : if, instead of taking $\Delta \lambda=0.1 \mathrm{~nm}$, we consider $\Delta \lambda=1 \mathrm{~nm}$, computing times are significantly reduced. In this case, the subsequent reduction of the number of optimal colors negatively affects the sampling quality of the MacAdam limits.

Our algorithm consists of calculating the tristimulus value $Y$ from an optimal wavelength pair, within the interval fixed by $\Delta L^{*}$. The condition imposed for our algorithm for each optimal color type is described in the following equations and is outlined in Fig. 2:

$$
\text { Type 1:Y }=\frac{100}{\overline{\mathbf{y}} \cdot \mathbf{S}} \sum_{k=i}^{j} \bar{y}\left(\lambda_{k}\right) \cdot S\left(\lambda_{k}\right) \in\left[Y_{0}-\Delta Y, Y_{0}+\Delta Y\right] \text {, }
$$

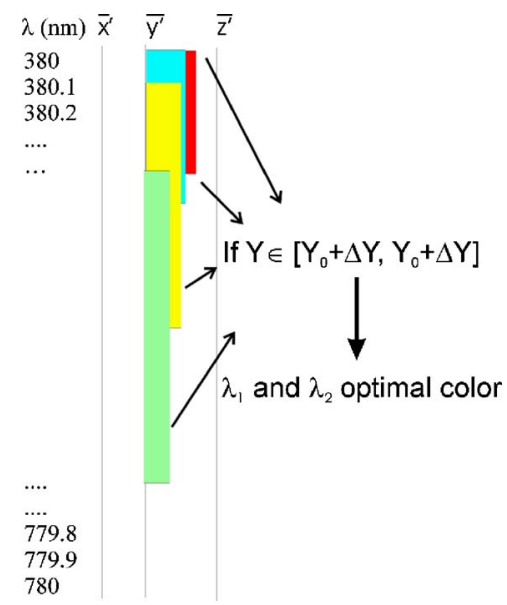

Fig. 2. (Color online) Scheme of our algorithm in column format. See text for more detail.

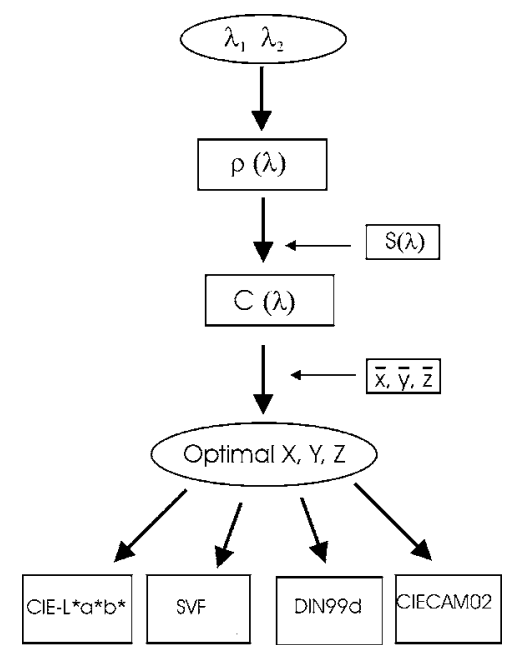

Fig. 3. General diagram for obtaining the color solid in several color spaces.
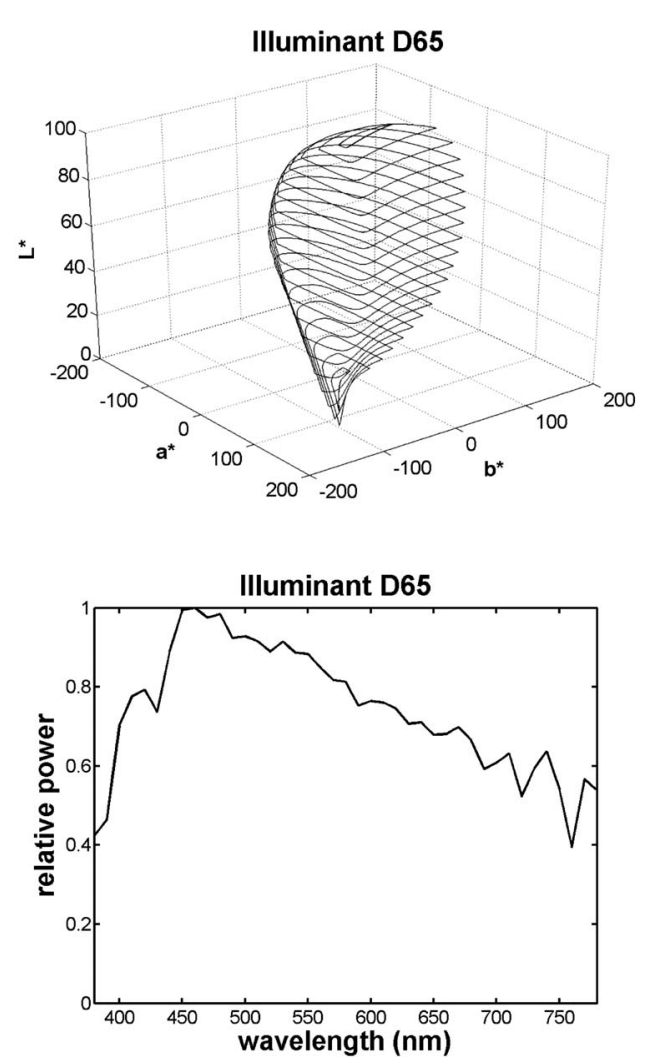

Fig. 4. Rösch-MacAdam color solid in the CIE- $L^{*} a^{*} b^{*}$ color space under the illuminant D65.

$$
\begin{aligned}
\text { Type } 2: Y & =\frac{100}{\overline{\mathbf{y}} \cdot \mathbf{S}}\left(\sum_{k=1}^{i} \bar{y}\left(\lambda_{k}\right) \cdot S\left(\lambda_{k}\right)+\sum_{k=j}^{N} \bar{y}\left(\lambda_{k}\right) \cdot S\left(\lambda_{k}\right)\right) \\
& \in\left[Y_{0}-\Delta Y, Y_{0}+\Delta Y\right]
\end{aligned}
$$

where $Y_{0}$ is the luminance factor calculated from the lightness value $L^{*}$ initially defined.

With each pair of limiting wavelengths, $\lambda_{1(=i)}$ and $\lambda_{2(=j)}$, and the illuminant-light source $S(\lambda)$ it is very easy to generate the optimal color stimuli $C_{\text {optimal }}(\lambda)$ as $\rho_{\text {optimal }}(\lambda)^{*} S(\lambda)$ with $N$ spectral samples. Obviously, from here one can almost immediately compute the $X Y Z$ tristimulus values from the color-matching functions and encode them into perceptual values in several color spaces (Fig. 3), such as CIELAB, SVF, DIN99d, and CIECAM02.

\section{RESULTS}

Since we initially calculated all the optimal colors of the color solid in the CIE- $L^{*} a^{*} b^{*}$ color space (Fig. 4), our algorithm returns optimal colors within a lightness interval. To compute the color solid in other color spaces, we have to consider that the model's equivalent variable to lightness need not be constant for all of the lightness data set. This happens, for instance, with the CIECAM02 color space, where the lightness $J$ depends on the achromatic response elicited by the stimulus. In any case, it should not be difficult to adapt the algorithm to the definition of lightness used in a particular model $(J$ in the CIECAM02 color space, $V$ in the SVF color space, $L_{99 d}$ in the DIN99d color space, etc.) 
We have uniformly sampled the ]0, 100[ lightness interval, at one-by-one steps. As can be seen in Table 1, for instance, our algorithm and the one originally due to MacAdam, ${ }^{4}$ with the same wavelength step $\Delta \lambda$, yield a considerably different number of optimal colors for several luminance factors under illuminant $\mathrm{C}$.

Another subject we bear in mind after the previous table is that the number of optimal colors obtained with our algorithm depends on the value of $L^{*}$ (Fig. 5), as well as on the tolerance $\Delta L^{*}$. If $\Delta L^{*}$ is very small, for example, smaller than 0.005 , the number of optimal colors will diminish considerably, so the smoothness of the plotted MacAdam loci will be reduced. But if this parameter is great, for example, greater than 0.5 , the number of optimal colors will be grouped in minicurves, the global representation of MacAdam loci will appear slightly stepped, and the graphic quality will decrease. Therefore, after testing several tolerance values we have found that $\Delta L^{*}$ $=0.01$ is optimal. This lightness tolerance value will guarantee that the MacAdam locus for each lightness plane is sufficiently smooth, rendering linear interpolation of these colorimetric data unnecessary.

\section{A. Color Solid in Constant Lightness Planes}

As we have just said, optimal color data associated with luminance factors lower than $10 \%$ and higher than $95 \%$ are not found in the literature. However, with our proposed method we can broaden the number of known optimal colors and find new ones for any luminance factor, from 0 to $100 \%$. Taking into account Eq. (1), we can search any optimal color for lightness values in the [1, 100] interval, at one-by-one steps.

After looking at the previous table, it is clear that with our algorithm we will be able to delimit the MacAdam loci more accurately than with the original MacAdam algorithm, without graphical interpolation, as can be seen in Fig. 4. However, the sampling of the optimal color loci made with our algorithm in each color space is not uniform, and it depends on the lightness value (Fig. 5). Independently of the color space or the illuminant/lamp selected, the MacAdam limits for high and low lightness values are better sampled than for intermediate lightness values, as can be seen in Fig. 5 .

As the lightness $L^{*}$ can be selected within the interval ]0, 100[, the complete figure of the color solid can be ob- tained for any illuminant or light source. Figure 6 shows the color solid in the CIE $L^{*} a^{*} b^{*}$ color space for three fluorescent illuminants: F2, F7, and F11. As can be seen, the shape of the color solid depends on the illuminant. We clearly can see that the shape of the color solid obtained with illuminant F11 is quite different from the rest. We also show in Fig. 7 the color solid for three real lamps ${ }^{19}$ : the standard high-pressure sodium lamp (HP1), the colorenhanced high-pressure sodium lamp (HP2), and the high-pressure metal halide lamp (HP3). As can be seen, the color solid for the HP1 lamp clearly differs from the rest. Therefore, perhaps it is possible to evaluate the color-rendering index of light sources from the number of distinguishable colors, estimated from the volume of its color solid. If this were possible, this colorimetric quality index for light sources would be absolute, without the necessity of taking a reference illuminant, such as the current CIE color-rendering algorithm ${ }^{20-23}$ proposes. This idea has been applied in a preliminary way by us in parallel with this work, and it will be summarized and discussed in the next section.

We have also calculated the color solid for the color spaces (Fig. 8), such as CIECAM02, DIN99, and SVF, since these color spaces are more uniform than CIE- $L^{*} a^{*} b^{*}$. We can see that these color solids are more

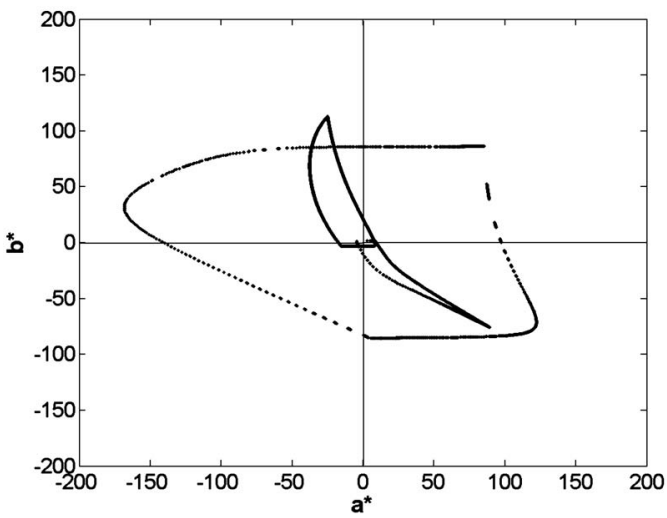

Fig. 5. Effect of the luminance factor $Y$ over the (calculated optimal color) symbol sampling of the MacAdam loci: the smaller MacAdam loci correspond to $L^{*}=1$ and $L^{*}=98$, while the larger one corresponds to $L^{*}=50$. It was clearly seen that in the larger locus the yellow-red quadrant is partially sampled, particularly for the red hues.

Table 1. Comparison between the Sampling of Optimal Colors, Using the Illuminant C and the CIE 1931 Standard Observer with the Same Spectral Sampling $(\Delta \lambda=0.1 \mathrm{~nm})$, Obtained with MacAdam's Algorithm ${ }^{4}$ and Our Algorithm

\begin{tabular}{|c|c|c|c|c|c|}
\hline \multirow{3}{*}{$\begin{array}{c}Y \\
\text { (Rounded } \\
\text { Toward the } \\
\text { Nearest Integer) }\end{array}$} & \multirow[b]{3}{*}{$L^{*}$} & \multicolumn{4}{|c|}{ Number of Optimal Colors } \\
\hline & & \multicolumn{2}{|c|}{ MacAdam's Algorithm } & \multicolumn{2}{|c|}{ Our Proposal } \\
\hline & & Type 1 & Type 2 & Type 1 & Type 2 \\
\hline 1 & 9 & - & - & 1034 & 1051 \\
\hline 10 & 38 & 8 & 7 & 1383 & 891 \\
\hline 20 & 52 & 8 & 8 & 645 & 681 \\
\hline 50 & 76 & 12 & 12 & 814 & 919 \\
\hline 70 & 87 & 12 & 12 & 1090 & 1342 \\
\hline 90 & 96 & 8 & 11 & 3935 & 4320 \\
\hline
\end{tabular}



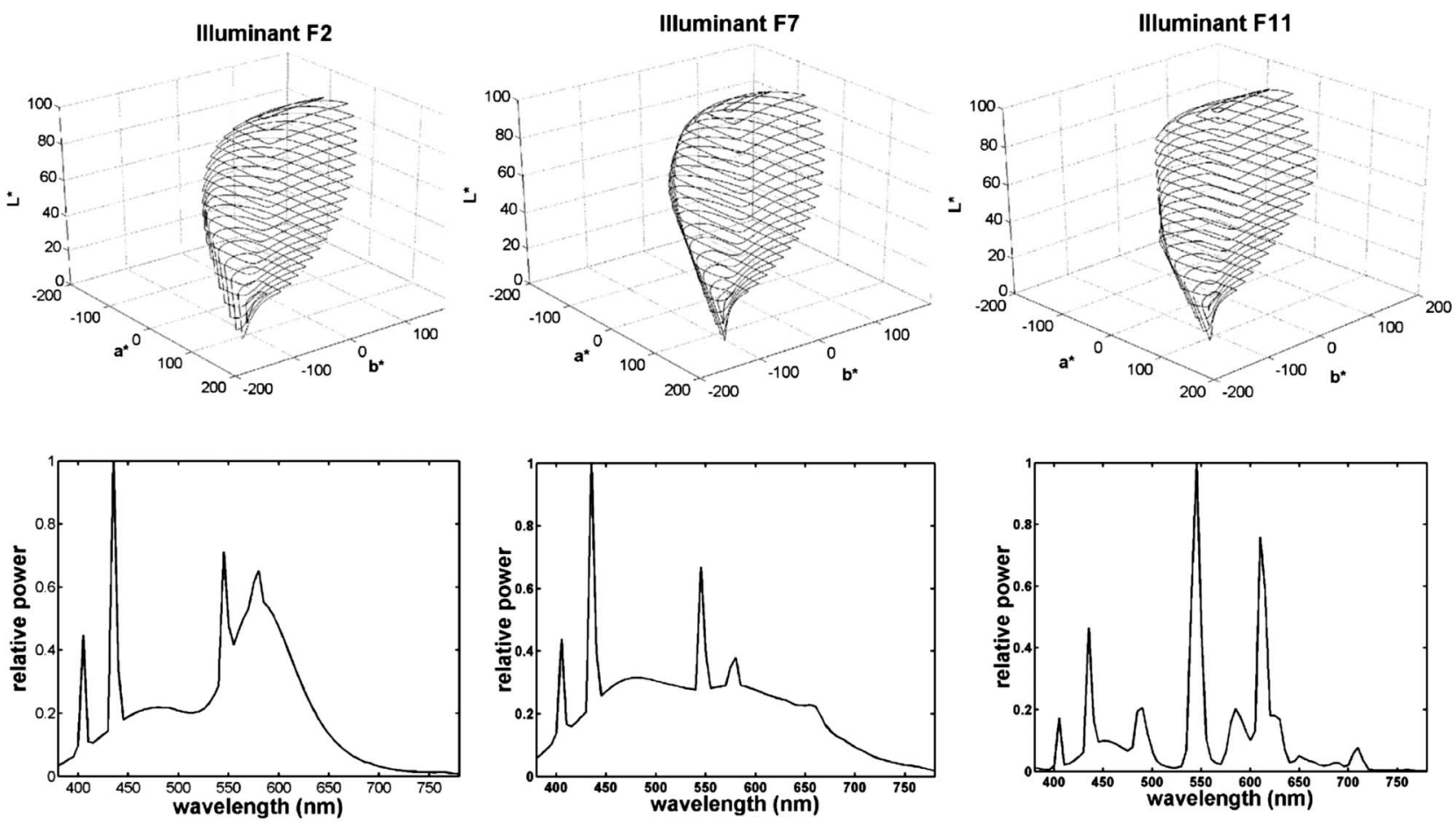

Fig. 6. Rösch-MacAdam color solid in the CIE- $L^{*} a^{*} b^{*}$ color space under three fluorescent illuminants: F2 (left), F7 (center), and F11 (right).
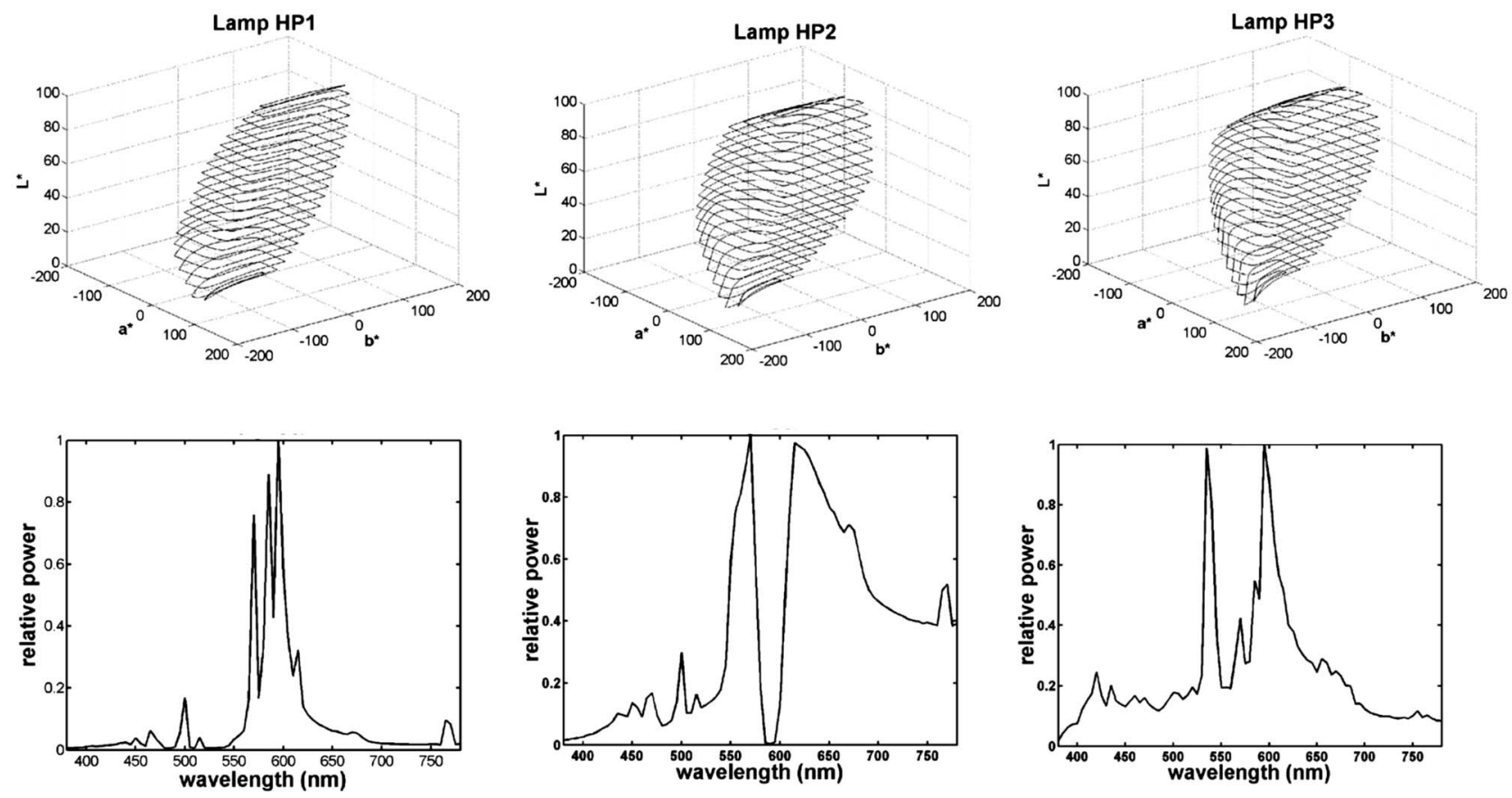

Fig. 7. Rösch-MacAdam color solid in the CIE- $L^{*} a^{*} b^{*}$ color space under three real lamps: HP1 (left), HP2 (center), and HP3 (right).

uniform, above all the color solids associated with the DIN99d and CIECAM02 color spaces because they look more spherical or homogeneous. The color solid in the SVF color space is not closed in the black vertex, unlike in the CIE- $L^{*} a^{*} b$ color space (Fig. 4 ) and the rest (Fig. 8). In the following section it will be discussed if the shape of the color solid ought really be more spherical or homoge- neous in uniform color spaces, such as CIECAM02 and DIN99d, or if this result is purely coincidental.

\section{B. Color Solid in Constant Hue-Angle Planes}

As we advanced in the Introduction, in this work we also show a method to plot the color solid in constant hue 
planes. The method basically consists of cutting up the color solid in vertical sections of constant hue. That is, we now try to draw the color solid $\left(a^{*}, b^{*}, L^{*}\right)$ in constant hue profiles with $C^{*}$ versus $L^{*}$ axes. To calculate the $a^{*}$ and $b^{*}$ coordinates, it is necessary to obtain previously the chroma as a function of the angle hue $h_{a b}{ }^{*}$, i.e., $h_{a b}{ }^{*}$ versus $C_{a b}{ }^{*}$, and we sometimes have to interpolate. In this case, although the curves are not very uniform, we have used the linear interpolation, since the spline interpolation does not show good results in the extreme values of the hue-angle interval. In future studies we will try to implement the Sprague interpolation algorithm proposed by the CIE. ${ }^{18}$ So, we take 120 hue-angle values, between 0 and 360, at intervals of $3 \mathrm{deg}$, since a change of $3 \mathrm{deg}$ ensures that all the different Munsell hues ${ }^{14}$ are considered, even in the SVF, DIN99d, and CIECAM02 color spaces. Therefore, we interpolate the $C^{*}$ value associated with the hue angle $h^{*}$; after that the $a^{*}$ and $b^{*}$ coordinates are computed, and finally the color solid is plotted. This procedure is the same for all the color spaces, except that in the other spaces used we work with the colorfulness and not with the chroma. For instance, in the CIECAM02 color space, ${ }^{13} M$ is the adequate variable, the corresponding cardinal coordinates are $a_{M}$ and $b_{M}$, and the hue angle is defined in a conventional way from these cardinal coordinates.

The color solids for the different color spaces for illuminants D65 and F11 and the real lamp HP1 are shown in Fig. 9, where we can also see the differences in the shape of the color solid, as we already saw in the color solid when it was plotted in constant lightness planes. If we represent graphically the complete color solid, the differences due to the illuminant are very subtle (Fig. 9). So, a more compact visualization (Fig. 10) allows us to compare simultaneously different color solids under different illuminants and light sources to analyze these differences better.

In Fig. 10, using different constant hue profiles in CIELAB associated with the primary Munsell hues, two continuous-spectrum illuminants, E and D65, are compared with a discontinuous-spectrum illuminant, F11, and a real light source, HP1, with a very peaked spectrum, spanning correlated color temperatures from approximately 2000 to $6500 \mathrm{~K}$. Again, we can see greater differences among these illuminants in the following hue regions: purple $(5 \mathrm{P})$, blue-green $(5 \mathrm{BG})$, green $(5 \mathrm{G})$, and blue-purple (5PB).
However, these comparisons made in CIELAB are quite preliminary, and not completely effective, because we are not applying a common chromatic adaptation to the optimal color data in order to render the absolute colorimetric comparison, under the same reference illuminant. Among the uniform color spaces used in this work, with their corresponding variables $(C, V)$ for $\mathrm{SVF},\left(C_{99}, L_{99}\right)$ for DIN99d, and $(M, J)$ for CIECAM02, only the last one has an embedded chromatic adaptation transform, CAT02, which can be simultaneously applied to all previous data under the same internal or cortical illuminant. So, we have calculated the corresponding colors of the previous MacAdam loci under each illuminant-lamp by means of the CAT02 transform ${ }^{13}$ (degree of adaptation or $D$ factor calculated by default, $D=0.9119$ ) in order to make simultaneously comparisons among them under the same illuminant. The white point of this reference illuminant lies between the illuminants $\mathrm{E}$ and D65. Figure 11 shows these calculations in CIECAM02 with constant hue profiles for the illuminants E, D65, and F11 and the real lamp HP1. Unlike Fig. 10, the greater similarities of color gamut among them are in the hue profiles $5 \mathrm{P}, 5 \mathrm{~B}$, and $5 \mathrm{~PB}$.

Making comparisons between pairs of illuminants in Fig. 11, and beginning with the E-D65 pair, it can be clearly seen in hue profiles $5 \mathrm{GY}, 5 \mathrm{G}$, and $5 \mathrm{BG}$ that, for very pale and light colors, some distinguishable colors under illuminant $\mathrm{E}$ can exist without equivalent chromatic appearance under illuminant D65. That is, if we consider chromatic adaptation to our reference illuminant, the gamut for very light colors for the illuminant $\mathrm{E}$ in this hue region is higher than that of the illuminant D65. But, in hue region $5 \mathrm{Y}$, the gamut of very light colors for the illuminant D65 is higher that associated with the illuminant E. This superiority in color gamut of D65 over E in color gamut is more evident in the increase of bright, strong, and deep colors, never perceptible under illuminant $\mathrm{E}$, in the hue regions $5 \mathrm{RP}, 5 \mathrm{R}$, and 5YR. This interesting analysis of perceptible color gamuts under both illuminants can be extended to more hue regions and with more graphic detail.

Proceeding analogously with the D65-F11 pair, again it can be clearly seen in Fig. 11 that we can perceive very light and bright greenish colors under illuminant F11 (along the range 5GY-5G-5BG) without perceptual correspondence under illuminant D65. In contrast, in the same hue regions, the perceptible color gamut under illuminant
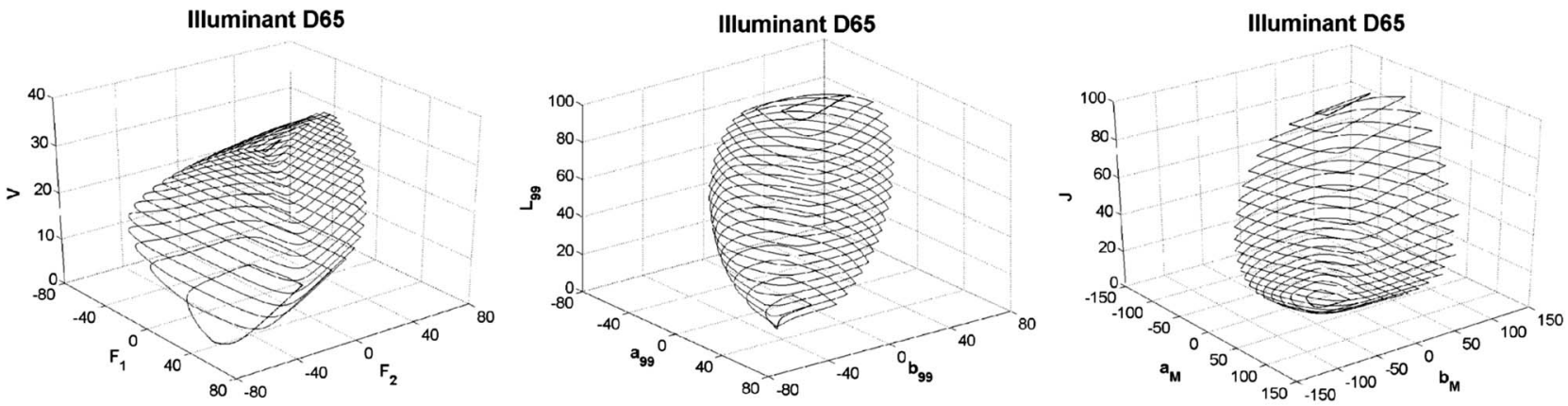

Fig. 8. Rösch-MacAdam color solids for the CIE 1931 standard observer under the illuminant D65 in different perceptual color spaces: SVF (left), DIN99d (center), and CIECAM02 (right). 


\section{CIE- L*a*b*}
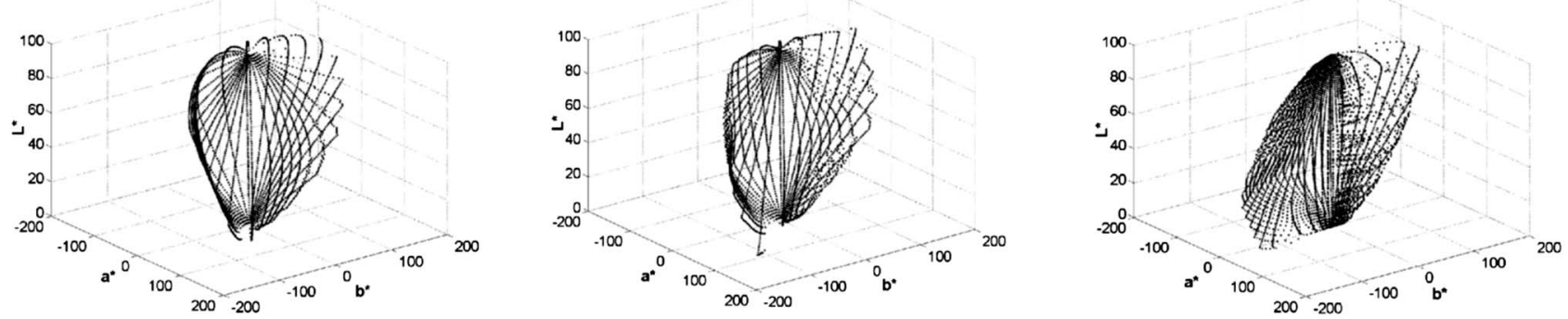

\section{SVF}
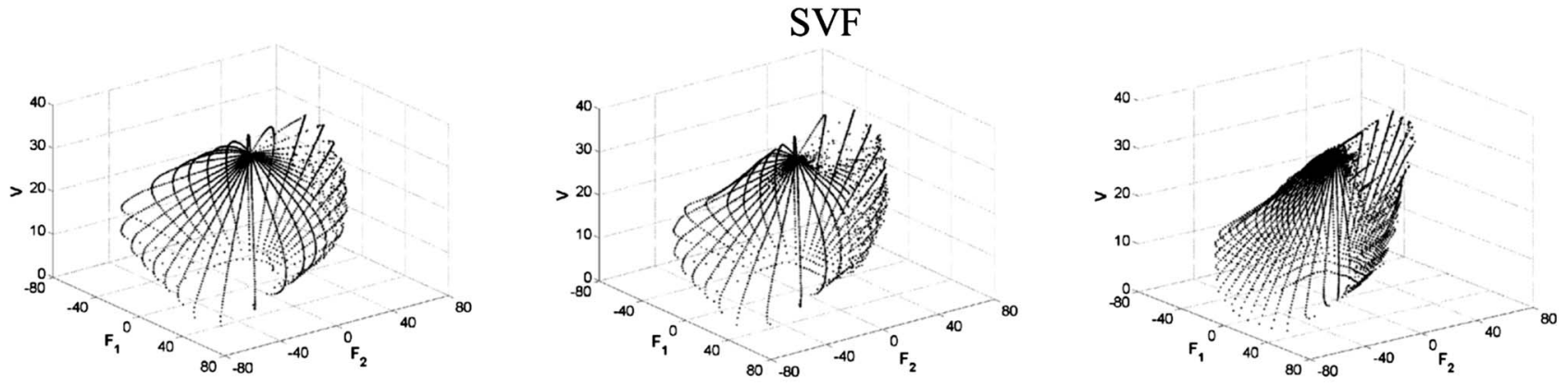

DIN99d
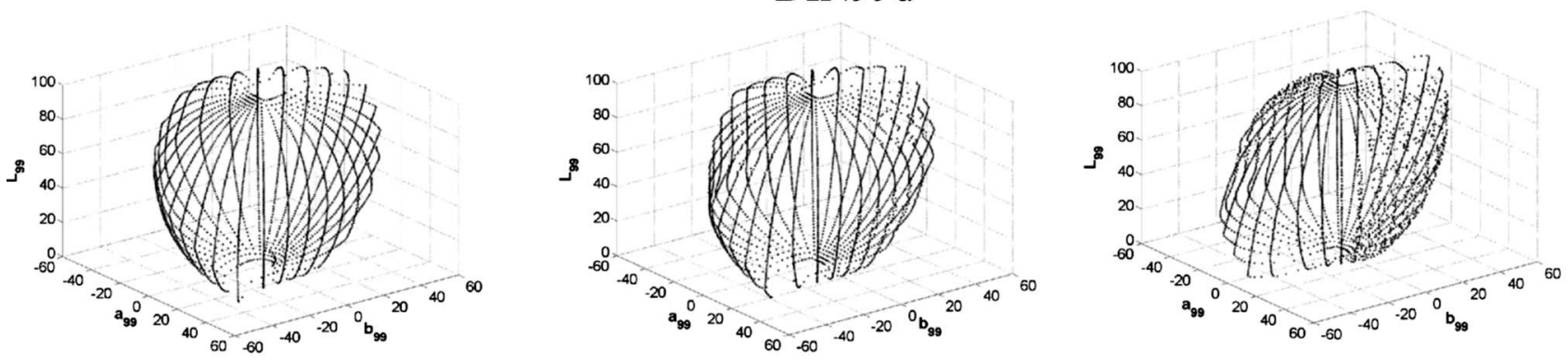

\section{CIECAM02}
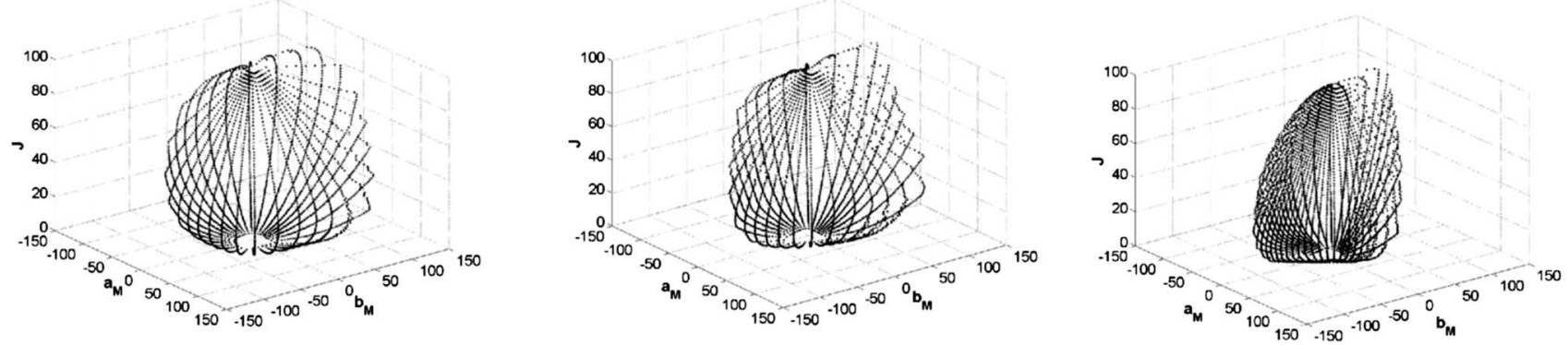

Fig. 9. Rösch-MacAdam color solid under the illuminants D65 (left) and F11 (center) and the real lamp HP1 (right) in the color spaces CIE- $L^{*} a^{*} b^{*}, \mathrm{SVF}$, DIN99d, and CIECAM02. Sixty hue profiles have been taken to avoid aliasing.

D65 is clearly higher than that of the illuminant F11 for strong and deep greenish colors. Similar analyses, and with more graphic detail, could be done in other hue regions (reds, oranges, etc.), but it is clear that the perceptible color gamut of the illuminant D65 is greater than that of the illuminant F11. Nevertheless, from the previous analysis it can also be inferred that, although the number of discernible colors under illuminant D65, if it might be obtained exactly, would be higher than that associated with the illuminant F11, not all perceptible colors under illuminant F11 do necessarily belong to the set of distinguishable colors of the illuminant D65 and vice versa.

Regarding the comparison HP1 versus D65, it is clear 
that the color gamut under illuminant HP1, and with color correspondence under illuminant D65, is inside the color gamut of illuminant D65 for most hue regions, except for the hue region $5 \mathrm{~PB}$, specifically in the region of very dark and deep colors.

Finally, similar comparisons can be done with other illuminant-lamp pairs in Fig. 11, as, for example, E versus F11 and HP1 versus F11. But, definitively, it can clearly be seen that, when the same color correspondence over the color solids is applied under different illuminants-lamps, the greatest perceptible color gamuts are for the illuminants E and D65 and the lowest one is for the lamp HP1. In contrast, we have found that there can be perceptible colors under one illuminant-light source without chromatic correspondence under other illuminants-light sources. In the next section, we discuss jointly all the results described up to this point, which could perhaps also be enlarged in future works.

\section{DISCUSSION}

In previous sections we have shown that the recalculation of optimal colors under several illuminants and light sources, encoded and three-dimensionally plotted as a color solid in several color spaces, has brought about some results and ideas that are worthwhile to recap and elaborate a bit more, even with additional results, because new
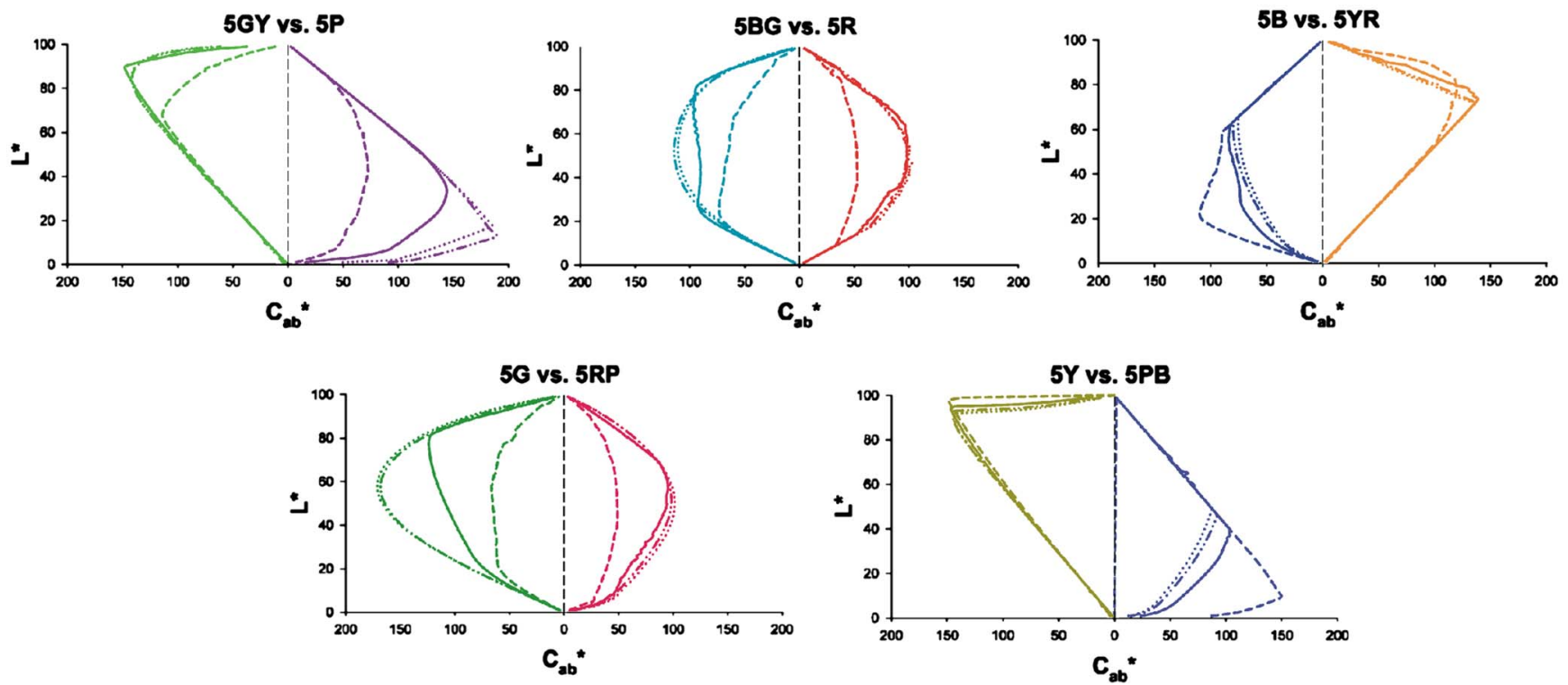

Fig. 10. (Color online) Constant hue-angle profiles of the Rösch-MacAdam color solid under several illuminants in the CIE- $L^{*} a^{*} b^{*}$ $\left(C_{a b}{ }^{*}, L^{*}\right)$ diagram (illuminant F11: solid curve; HP1: dashed curve; E: dotted curve; and D65: dashed-dotted-dotted curve).
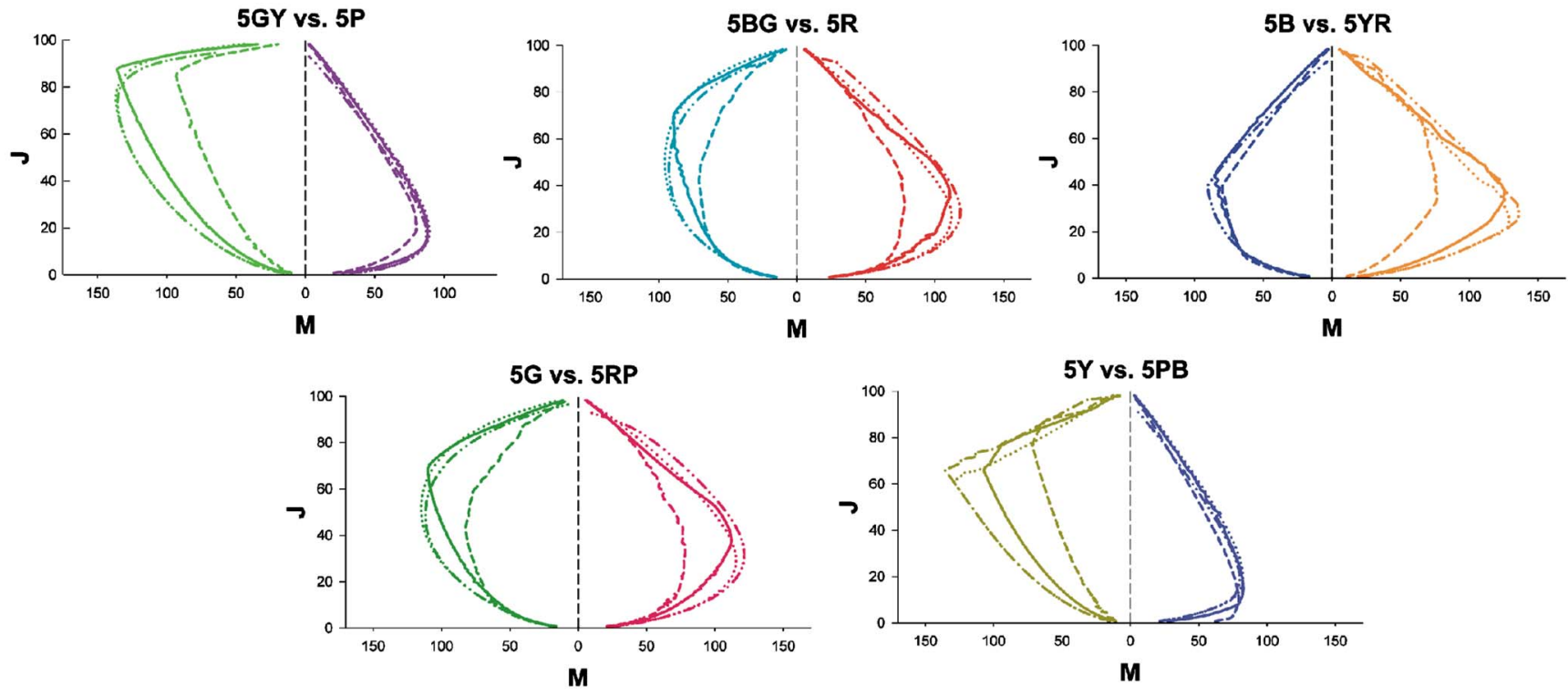

Fig. 11. (Color online) Constant hue-angle profiles of the Rösch-MacAdam color solid under several illuminants in the CIECAM02 $(M, J)$ diagram (illuminant F11: solid curve; HP1: dashed curve; E: dotted curve; and D65: dashed-dotted-dotted curve). 
interesting works about colorimetry of light sources and color perception can be separately derived from this work.

\section{A. Concerning the Shape of the Color Solid in a Uniform Color Space}

The three-dimensional plot of the color solid in several color spaces (Figs. 8 and 9), some of them quite uniform, gives rise to the question of whether the shape of a color solid in a perfectly uniform color space should be completely spherical. Therefore, this subject is also linked with a comparative analysis of the uniformity of the color spaces used in this work. It was clear until now that the DIN99d and CIECAM02 spaces are the most uniform ones because they were designed with the aim of improving the uniformity of older color spaces (CIELAB, SVF, etc). Due to this reason, as can be seen in Figs. 8 and 9, the color solids under any illuminant-lamp look more spherical or homogeneous in CIECAM02 and in DIN99d than in CIELAB or SVF. We might conclude that the CIECAM02 color space is a bit more uniform than the DIN99d color space. In fact, as this last one is based on the CIELAB color space, which has enough uniform defects in the purple region, particularly for very dark colors, a protuberance, but less emphasized, can still be seen in Figs. 8 (center) and 9 (right) for dark and deep purple colors. Therefore, we can conclude that the CIECAM02 color space, due to its uniformity, encodes the color solid with greater homogeneity. However, this does not mean necessarily that in a hypothetically perfectly uniform color space the color solid under illuminate D65, for instance, will be a perfect sphere. Taking Figs. 8, 9, and 11 into account, it is clear that the shape of the color solid under illuminant D65, or E, is rounded but not a perfect sphere. This is caused by the spectral tuning of the illuminant-lamp spectrum with the color-matching functions, above all with $\bar{y}(\lambda)$ and the luminous efficiency curve $V(\lambda)$, that is, by the shape and area of the product $S(\lambda) \cdot V(\lambda)$. Indeed, as the original MacAdam's works ${ }^{4,5}$ proved and as can be seen in classical colorimetry textbooks, the greater variety of light colors appear in the green-yellow (GY) hue region. Due to this reason, taking into account the approach of the constant lightness profiles, the snap of the MacAdam loci toward the absolute white is not homogeneous or circular. As is easily seen in Figs. 4-8, there is always a protuberance on the upper level of all the color solids, independently of the illuminants/lamps and/or color spaces used, but displaced from green to orange depending on the correlated color temperature (chromaticity) of the illuminant-lamp. Consequently, it is not a necessary condition that the color solid encoded by the most uniform color space should be plotted as a perfect sphere. For this reason, the Munsell color tree is clearly asymmetric, with longer branches present at high Munsell values for (typically) yellowish hues and at low Munsell values for (typically) purple hues. In the case of the CIECAM02 color space, the snap of the color solid toward the absolute black will be always more homogeneous or circular than that toward the absolute white. Therefore, the MacAdam limits with very high lightness never will be homogeneous or circular, except in a quasi-triangle region around the yellowness perceptual axes. One further work to be devel- oped from this issue would be to use, as a reference, the color solid corresponding to the perceptually determined data of the Munsell Renotation System (or a similar data set, for instance, Natural Color System data) with extrapolated specification for the optimal colors ${ }^{24,25}$ and to determine how much the color solids in question depart from this.

On the other hand, applying CIECAM02, we have found that the shape of the color solid can change significantly according to the degree of chromatic adaptation or factor $D$. In the above figures associated with CIECAM02, this color model has been always applied by calculating by default the corresponding factor $D(=0.919)$. But, testing other $D$ values, for instance, $D=0$ (without adaptation) and $D=1$ (complete adaptation), the shapes of the color solid for the same illuminant-lamp can be very different. In Fig. 12 the color solids at constant lightness planes for three illuminantslamps (HP1, F11, and D65) are shown, visualized from top to bottom, for two degrees of adaptation $(D=0$ and $D=1)$. As we can see, the color solids varying less are those associated with the illuminant D65, while those varying more belong to the lamp HP1. Color solids associated with the illuminant $\mathrm{E}$ with this new test have not been plotted because their changes were minimal with both adaptation conditions, so this reinforces the fact that the cortical or internal illuminant for the CIECAM02 color model is nearer in chromaticity to the illuminant $\mathrm{E}$ than to the illuminant D65. Taking the last figure into account, since human color perception always works with chromatic adaptation, in a lesser or greater degree, but never with $D=0$, the right side of Fig. 12 represents an alternative way of viewing in a perceptual color space, without chromatic adaptation, the MacAdam limits shown for many years in chromaticity diagrams ${ }^{8-10}$ as CIE- $x y$, CIE- $u^{\prime} v^{\prime}$, etc. As can be clearly seen in this figure, the HP1 data indicate a strong colorimetric shift toward the orange region of solid color (the equal-energy white stimulus would be perceived as orange) due to the chromaticity (color temperature) of the light source, above all for very light colors. This accentuated colorimetric shift is partially neutralized by chromatic adaptation, which serves to justify the perceptual phenomenon of color constancy.

However, regarding the cases with complete chromatic adaptation, which are the ones most similar to the factor $D$ calculated by default in the previous section, it can also be seen in Fig. 12 and likewise in Fig. 11, if only the envelope of the constant lightness planes of color solids is taken into account, that there are perceptible colors under illuminant F11 in the green-yellow quadrant, with middle lightness, which are not perceptible under illuminant D65, not even under lamp HP1. Furthermore, from the top view, it seems that the region covered by the D65 color solid is altogether greater than that of the illuminant F11. So, this result again proves that there can be perceptible colors under one illuminant/light source without perceptual correspondence under another illuminantlight source, even if the first illuminant had in all a more limited number of discernible colors than the second illuminant.

On the other hand, it is worthwhile to discuss the non- 

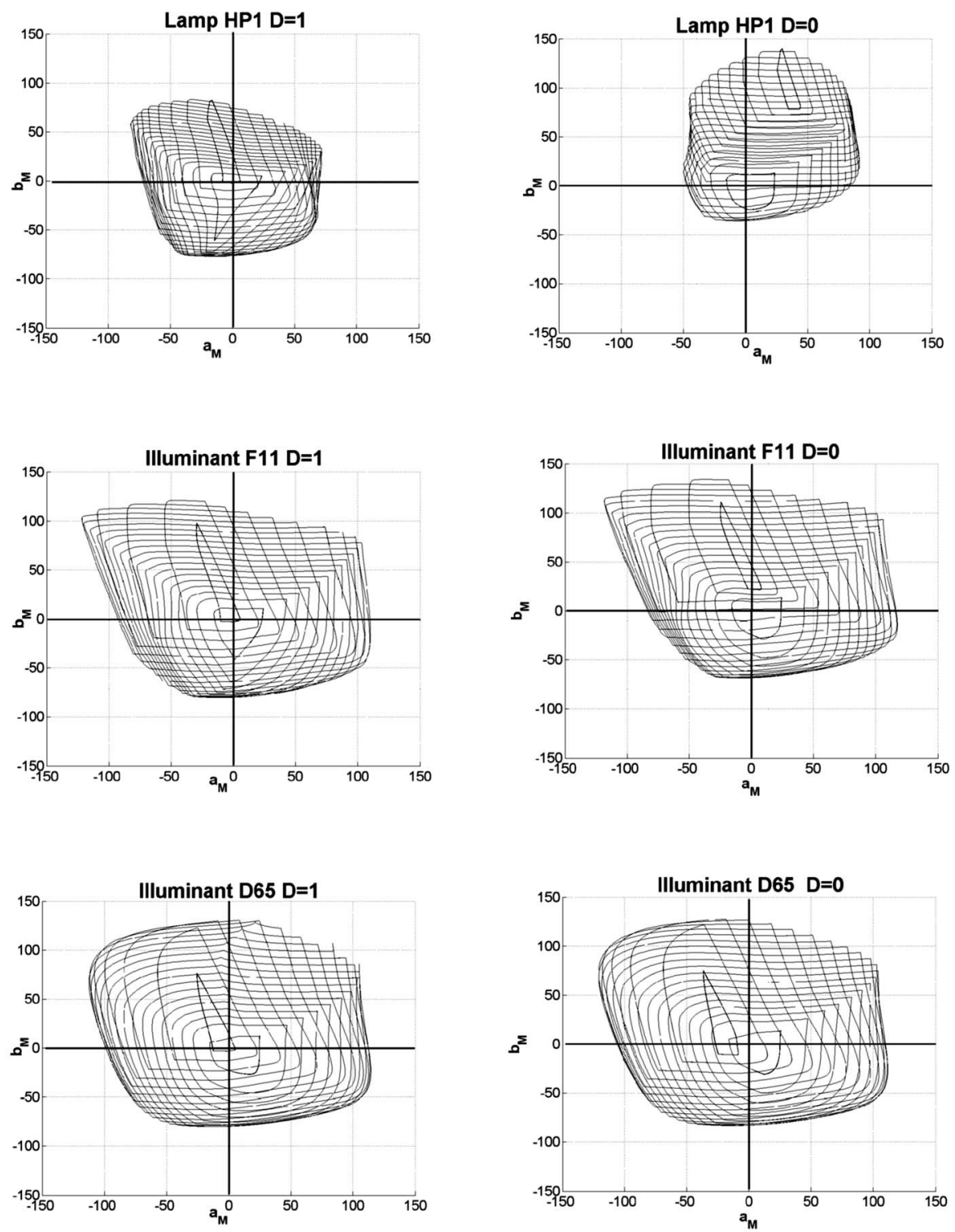

Fig. 12. Top view of some color solids under several illuminants-lamps in the CIECAM02 color space with different chromatic adaptation degrees (left: $D=1$; right: $D=0$ ). (The MacAdam locus with the solid curve corresponds to the highest constant lightness plane.)

uniformity of the color solid toward the black when it is encoded by SVF color space. Despite the fact that this color space was designed with the aim of plotting uniformly the constant lightness Munsell loci, the mathematics of this color model causes the corresponding color solid not to taper homogeneously toward the absolute black, unlike what happens toward the absolute white (Figs. 8 and 9). Since this color model is fitted to the Munsell Atlas, its equations include a threshold value $\left(S_{0}=0.43\right)$ in order to adapt the scaling of the Munsell value. So, for $Y$ values lower than $0.43 \%\left(L^{*}<3.88\right)$, all optimal colors with lower lightness are encoded with value $V_{\mathrm{SVF}}=0$. This colorimetric behavior is common in any physiological color space, with a threshold value below which the response is zero, not negative. Due to this, the color solid plotted in this color space does not taper toward a point (absolute black), since there is a gap between the last graphed MacAdam locus and the absolute black. Thus, the cutoff in the lowermost part of the color solid in the
SVF color space is in agreement with the physiological premises imposed. In spite of this, it seems adequate to improve the modeling of the SVF color space for very dark colors.

\section{B. Concerning the Content of the Color Solid according to Different Light Sources}

The changes in the shape of the color solid with the spectral content of the illuminant-light source raise the issue of the differences in colorimetric quality among illuminants and light sources. This topic will be analyzed in more detail in Subsection 4.C, but it is also connected with the questions of how many color sensations, with a given illuminant-light source, we can distinguish and why this depends on its spectrum and its chromaticity. To understand this matter better, it is necessary to make simultaneous comparisons of color solids under several illuminants-lamps but taking chromatic adaptation into 
account, for instance, using the CAT02 transform of the CIECAM02 color appearance model. For this reason, Fig. 11 , obtained with CIECAM02, is a correct representation of the color solid (with constant hue profiles) under the same perceptual correspondence, that is, under the reference or internal (cortical) illuminant of the human visual system, similar in chromaticity to the equienergetic illuminant. From this figure, and all cited above, we may conclude the following:

- The color solids associated with illuminants-lamps, with correlated color temperature $T_{C}$ lower than $5500 \mathrm{~K}$, will have color gamuts smaller than those associated with illuminants-lamps with $T_{C}$ equal or higher than $5500 \mathrm{~K}$. This is clearly proved for the lamp HP1, often used in urban lighting, and the fluorescent illuminant F11, often used in interior lighting. It is a pending matter whether in a higher range of color temperature there is an upper limit above which the color gamut will diminish. To test this, we have also calculated the color solid corresponding
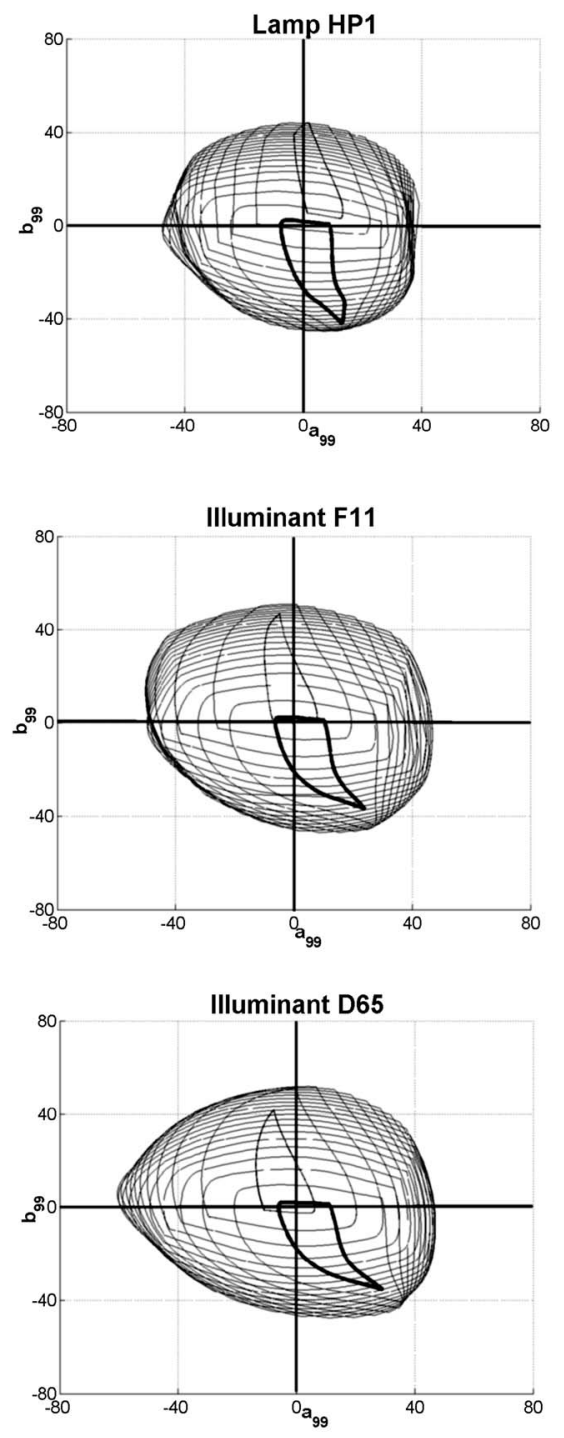

Fig. 13. Top view of several color solids under several illuminants/lamps, with the same color correspondence to illuminant D65, in the DIN99d color space. From top to bottom and from left to right: lamp HP1 $\left(T_{C}=1960 \mathrm{~K}\right)$, illuminant A $\left(T_{C}=2856 \mathrm{~K}\right)$, illuminant F11 $\left(T_{C}=4000 \mathrm{~K}\right)$, illuminant $\mathrm{E}\left(T_{C}=5500 \mathrm{~K}\right)$, illuminant D65 $\left(T_{C}=6500 \mathrm{~K}\right)$, and illuminant D100 $\left(T_{C}=10,000 \mathrm{~K}\right)$. (The MacAdam locus with the solid curve corresponds to the lowest constant lightness plane.)

to the illuminant D100, with $T_{C}=10,000 \mathrm{~K}$, and it has been plotted in the DIN99d color space by applying previously on the optimal $X Y Z$ data the CAT02 transform for the illuminant D65, as was done for the lamp HP1 and the illuminants A, F11, E, and D65, with the aim of covering roughly a large chromaticity range. We now have used the DIN99d color space because it is more coherent to show corresponding colors in a color space different from that associated with a one-color appearance model (CIECAM02), which describes and applies a chromatic adaptation transform (CAT02). A top view (Fig. 13) of the MacAdam loci associated with these illuminants-lamps supports what we said above: for $T_{C}<5500 \mathrm{~K}$, the lower the correlated color temperature, the smaller the distinguishable color gamut. But, in contrast, we have not found a similar behavior for $T_{C}$ higher than $10,000 \mathrm{~K}$, although is it possible that from any point of the $[10,000$, $+\infty$ ] K range the reduction of the gamut volume again turns up. However, this preliminary corollary cannot be
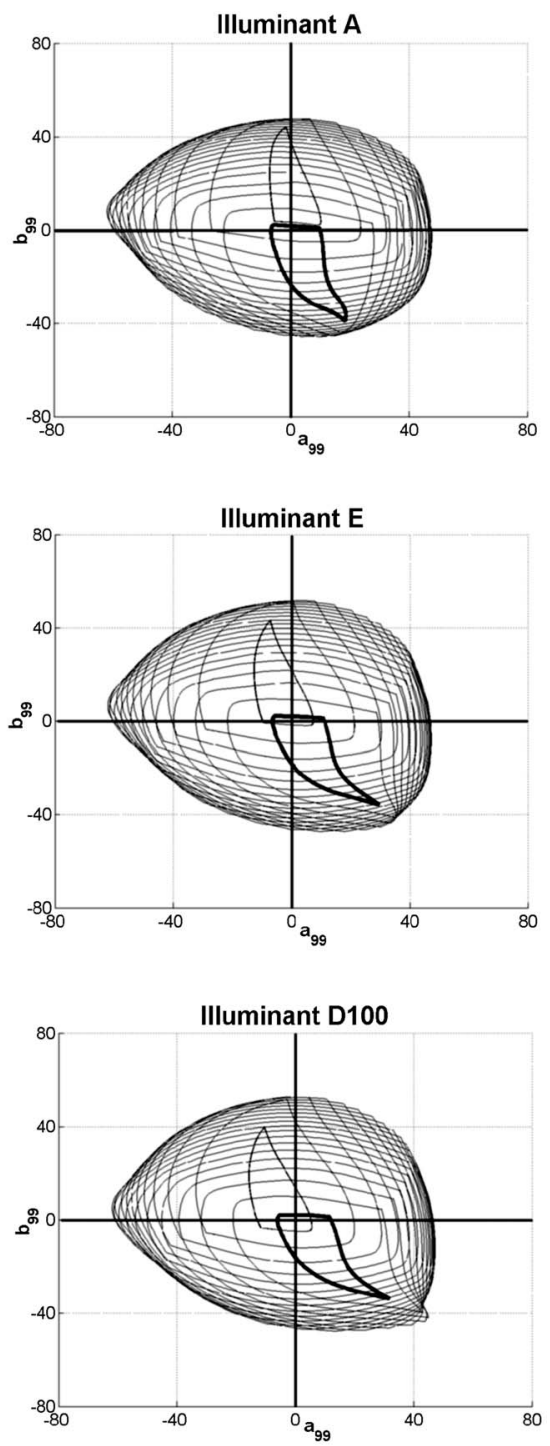


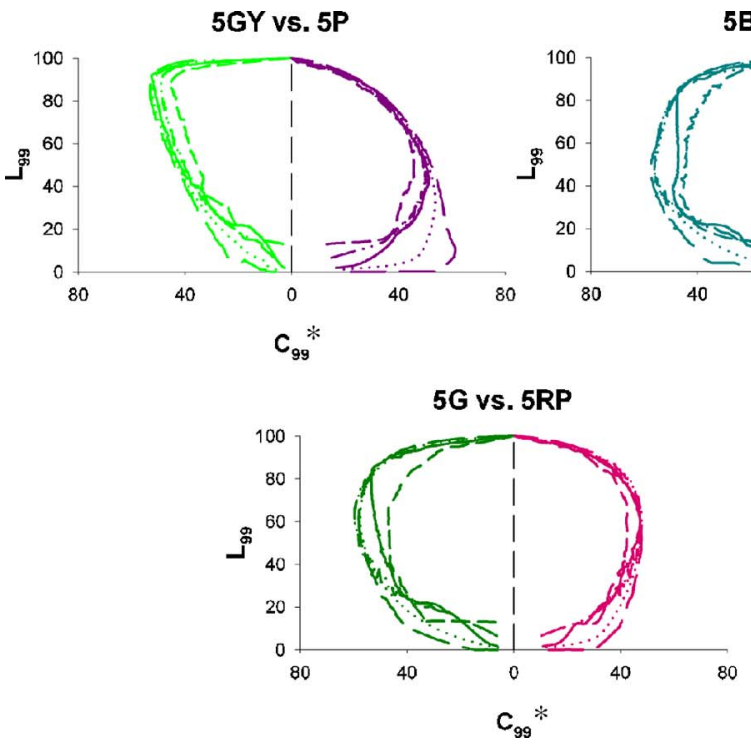

5BG vs. $5 R$

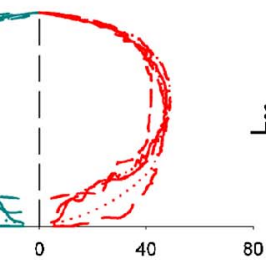

$\mathrm{C}_{99} *$

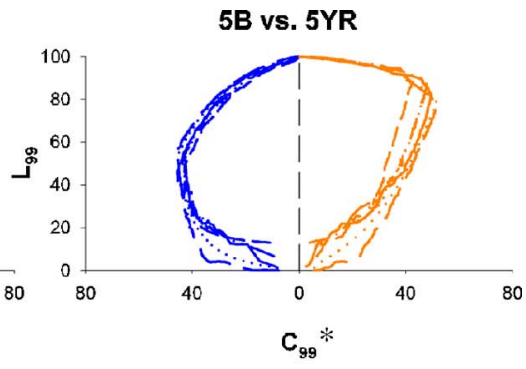

$5 Y$ vs. 5PB

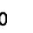

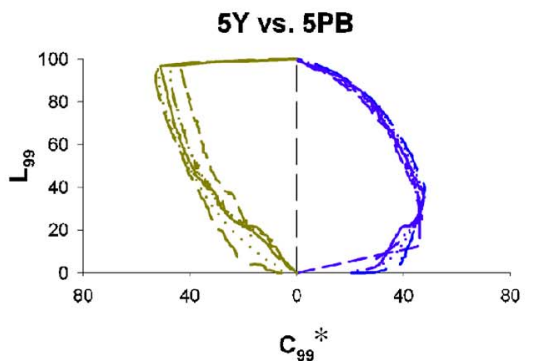

Fig. 14. (Color online) Constant hue-angle profiles of the Rösch-MacAdam color solid under several illuminants, with the same color correspondence to illuminant D65, in the DIN99d $\left(C_{99}, L_{99}\right)$ diagram (illuminant F11: solid curve; HP1: short-dashed curve; D65: dotted curve; A: dashed-dotted-dotted curve; and D100: long-dashed curve).

correct for all very narrowband lamps, for instance, twoband lamps, real or simulated. Hence, further research should be done on this issue.

- Taking into account the analyses derived from Figs. 11, 13 , and 14, we have found that there are color sensations under one illuminant-lamp, near the MacAdam limits, which do not have perceptual correspondence under other illuminants-lamps. But, moreover, we have proved that there can be illuminants-lamps with reduced color gamuts in comparison with other illuminant-gamut pairs, which have a small but significant number of color sensations imperceptible under those illuminants/lamps with greater color gamuts. Therefore, these conclusions imply that the number of colors discernible by the human visual system can be unlimited ${ }^{26}$ because it needs not be associated with only one illuminant-lamp, but, regarding the variety of natural or artificial light sources, we can predict and verify new color sensations that do not correspond with known illuminants/lamps. Some examples about this involving some hue regions have been given in Fig. 11 and can be again given in Figs. 13 and 14, above all with the aim of comparing the illuminants A and D100 relative to the illuminant D65. Nevertheless, perhaps this preliminary analysis and its conclusions might be enlarged with more graphic detail, and with greater statistical diversity of illuminants and lamps, in a future work because this would be very interesting for the lighting community, for its applications (museums, sport, and arts entertainments, etc.), and for the CIE.

- Regarding the quantity and variety of colors distinguishable by the human visual system and taking the results shown in Fig. 12 into account, where the shape and volume of the color solids are compared as a function of the degree of chromatic adaptation, it is clear that if our visual system did not use chromatic adaptation the number of discernible colors would be much greater, with minor perceptual correspondence among illuminants and light sources. Therefore, the color constancy phenomenon, ${ }^{27-31}$ based at the first stages on a chromatic adaptation transform, means from a evolutionary point of view an adaptive mechanism to reduce the variability of perceptually noncorresponding distinguishable colors. Thanks to this adaptive mechanism, the result of the evolution of several million years, our primate predecessors achieved a quasi-invariant system for encoding color in front of chromaticity changes of ambient light, clearly advantageous for establishing quasi-constant recognition patterns of objects and scenes very important for survival.

\section{Concerning the Proposal of an Absolute Colorimetric Index of Illuminants and Light Sources Based on the Volume of the Color Gamut}

As we said above, it is clear that the shape and volume of the color solid depends on the spectral content of the illuminant-light source. Therefore, we may say that the greater the gamut volume, the greater the number of distinguishable colors. Then we could propose a colorimetric quality index based on this condition for classifying any illuminant/lamp. This colorimetric quality index among light sources would be absolute, with no need for using a reference illuminant, such as the current CIE colorrendering algorithm proposes. Therefore, what we need is to find one or several methods for calculating the gamut volume involving directly, for instance, the estimation of the total number of distinguishable colors inside the color solid. In a preliminary work, ${ }^{32}$ done by ourselves, several methods for calculating the number of distinguishable colors inside the color solid were tested (Table 2). The first method consists of computing the partial counts of distinguishable colors for each constant lightness MacAdam locus encoded by CIECAM02, with a lightness step $\Delta L^{*}=1$, from 1 to 100, by a squares-packing method with unity area, without overlap, inside each MacAdam locus. In this way, the sum of these partial counts from $L^{*}=1$ to $L^{*}$ $=100$ gives the total number of distinguishable colors under each illuminant/light source. An alternative method, which gives similar results, consists of using the convex 
hull function by MATLAB for constant lightness MacAdam loci. The third method is named the ellipses-packing method. This takes Krauskopf and Gegenfurtner's discrimination model, ${ }^{33}$ based on psychophysical data into account and works by filling the constant lightness MacAdam loci, previously transformed by the CAT02 transform under illuminant $\mathrm{E}$, with discrimination ellipses increasing in area with increasing distance from the achromatic point in a modified MacLeod-Boynton chromaticity diagram. Consequently, again accumulating the partial counts of nonoverlapped ellipses or distinguishable colors for each constant lightness MacAdam locus from $L^{*} 1$ to $L^{*} 100$, with lightness step $\Delta L^{*}=1$, we can estimate the total number of distinguishable colors inside the color solid for any light source.

In Table 2 we show the preliminary results applying these three packing methods for several illuminants (A, C, D65, E, F2, F7, F11) and lamps (HP1-HP3), and they are compared with the standard CIE color-rendering algorithm. ${ }^{20,21}$ The three packing methods give absolute results of colorimetric quality, since they do not depend on a reference illuminant, as the current CIE algorithm does. However, despite the fact that there is a very good correlation in the ordering scale of quality in the three methods relative to the CIE algorithm, the magnitude of the numerical results in the three methods is very different. Thus, we might say that in applying the squarespacking (convex hull) method the number of distinguishable colors under each illuminant/lamp is estimated by excess. In contrast, in applying the ellipses-packing method, the number of distinguishable colors is estimated by defect. Therefore, we think that much more work is needed, for instance, testing a spheres-packing method in the most uniform color space available, in order to make a careful study of this subject concerning the colorimetry of light sources and color perception.

However, these methods and their preliminary results could be useful to develop new applications in color imaging, as, for instance, comparing the color gamuts of color devices, ${ }^{34-36}$ and in lighting design (museums, sports, TV, cinema, etc.). This could even be applied in order to evaluate the distinguishable colors of animal vision, such as dichromatic, trichromatic, or higherdimensionality vision, provided that the internal model of chromatic discrimination and encoding was known for each species.

On the other hand, it could be highly questionable if the gamut volume alone is suitable as a quality index in this sense. When not also taking the shape of the color solid into account, the gamut volume may say little about color-rendering capabilities. Thus, the great challenge should be to find a parameter that depends on both volume and shape. For instance, and to illustrate this, one can imagine two (theoretical) color gamuts, one represented by a spherical color solid centered at the midpoint of the achromatic axes and the other by a hemisphere of the same volume and with the achromatic axes coinciding with a diameter of its base. There is no question that the former should give the highest colorimetric quality index. Taking into account this and the preliminary results shown in this subsection (Table 2), it seems that the calculation of an absolute colorimetric quality index from knowledge of the color gamut volume and shape is far from trivial. In spite of this, one possible solution could be a calculation of an intersection volume of two color gamuts, $A$ and $B$ (this one as reference, for instance, as the illuminant $\mathrm{E}$ has been used in Table 2), as the average of $A \backslash$ (the intersection of $A$ and $B$ ) and $B \backslash$ (the intersection of $A$ and $B$ ). In contrast, this fact would mean a return to the relative colorimetric index of illuminants and light sources. But, at least, all illuminants and real lamps would be normalized by the same reference illuminant, so it could be considered as a common (absolute) colorimetric index.

\section{CONCLUSIONS}

In this work we have improved the algorithm for calculating the optimal colors proposed originally by MacAdam. The algorithm can be applied at any lightness value, so the color solid can be well sampled either in constant lightness planes or in constant hue planes for any color space. Unlike the irregularly shaped color solid obtained in CIELAB, the color solids associated with the most cur-

Table 2. Total Number of the Distinguishable Colors under Several Illuminants and Light Sources according to Several Packing Methods of Constant Lightness MacAdam Loci

\begin{tabular}{|c|c|c|c|c|c|c|}
\hline $\begin{array}{c}\text { Light } \\
\text { Source Test }\end{array}$ & $\begin{array}{l}\text { Illuminant } \\
\text { Reference }\end{array}$ & $\begin{array}{l}\text { Ellipses } \\
\text { Method }\end{array}$ & $\begin{array}{l}\text { Squares } \\
\text { Method }\end{array}$ & $\begin{array}{c}\text { Convex } \\
\text { Hull Method }\end{array}$ & $R_{a}(\mathrm{CIE})$ & $\begin{array}{l}\text { Ranking } \\
\text { (Squares } \\
\text { Method) }\end{array}$ \\
\hline A & P2856 & 25,851 & $1.753 \mathrm{e} 6$ & $1.309 \mathrm{e} 6$ & 99.58 & 5 \\
\hline $\mathrm{C}$ & D65 & 33,500 & $2.046 \mathrm{e} 6$ & $2.072 \mathrm{e} 6$ & 97.39 & 2 \\
\hline D65 & D65 & 30,736 & $2.013 \mathrm{e} 6$ & $1.532 \mathrm{e} 6$ & 99.58 & 3 \\
\hline $\mathrm{E}$ & D55 & 30,274 & $2.050 \mathrm{e} 6$ & $2.044 \mathrm{e} 6$ & 95.11 & 1 \\
\hline F2 & $\mathrm{P} 4230$ & 26,323 & $1.665 \mathrm{e} 6$ & $1.652 \mathrm{e} 6$ & 62.83 & 7 \\
\hline F7 & D65 & 30,732 & $1.968 \mathrm{e} 6$ & $1.971 \mathrm{e} 6$ & 90.23 & 4 \\
\hline F11 & $\mathrm{P} 4000$ & 26,311 & $1.735 \mathrm{e} 6$ & $1.304 \mathrm{e} 6$ & 82.91 & 6 \\
\hline HP1 & P1960 & 22,465 & $1.050 \mathrm{e} 6$ & $0.770 \mathrm{e} 6$ & 8.29 & 10 \\
\hline HP2 & P2510 & 25,465 & $1.663 \mathrm{e} 6$ & $1.240 \mathrm{e} 6$ & 82.59 & 8 \\
\hline HP3 & P3140 & 25,492 & $1.661 \mathrm{e} 6$ & $1.649 \mathrm{e} 6$ & 82.50 & 9 \\
\hline
\end{tabular}


rent perceptual color spaces, DIN99d and CIECAM02, look more spherical or homogeneous. However, this result does not imply that the color solid should be perfectly spherical in an ideal uniform color space, as we discussed above. Despite this, we think it would be interesting to determine in the future whether some characteristic of the color solid could serve as a test of the uniformity of a color appearance model. In the case of the SVF model, a uniformity defect around very dark colors was found due to the mathematics of that color model.

Once the color solids for different illuminants and light sources are shown, it can be seen that their shape and content clearly depend on the associated illuminant. Therefore, we can also conclude that the number of distinguishable colors, evaluated as the gamut volume, could depend on the associated illuminant. Furthermore, some interesting corollaries have been derived from both main conclusions about the colorimetry of light sources and color perception, which would be interesting to extend more profoundly in the future:

- The color gamuts associated with illuminants/lamps, whose correlated color temperature $T_{C}$ was inside the $[5500,10,000] \mathrm{K}$ interval, are greater than those associated with illuminants/lamps with their $T_{C}$ outside the cited interval. If $T_{C}$ moves enough from $5500 \mathrm{~K}$, the gamut volume will diminish in a uniform color space. However, this preliminary corollary cannot be correct for very narrowband lamps, for instance, two-band lamps, real or simulated. Hence, further research should be done to elucidate this open question.

- Applying the same color correspondence among optimal color data for each illuminant/lamp, we have found that there are distinguishable colors under one illuminant/lamp without perceptual correspondence under other illuminants/lamps, even though its gamut volume was small. This means that the number of colors discernible by the human visual system is unlimited because it cannot be associated with a single illuminant/lamp, but, in accordance with the variety of natural and artificial light sources, we can predict and verify new color sensations that do not match those of other known illuminants/ lamps.

- An additional conclusion from above is that color constancy, based at the first stages on a chromatic adaptation transform, can be also understood as an adaptive mechanism reducing the diversity of distinguishable colors without common perceptual correspondence with multiple illuminants/lamps.

- Finally, it has been proved with these preliminary results that it is possible to define an absolute colorimetric quality index for any illuminant/light source, based on the computation of the number of distinguishable colors inside the color solid. This proposal could be used as an alternative method to the (relative) color-rendering algorithm $^{20}$ proposed by CIE. The proposed methods in these calculations could also be used to evaluate and compare color gamuts of color-imaging devices ${ }^{34-36}$ and even of other natural vision systems (dichromacy, trichromacy, etc.). However, it seems adequate to work more much in the future with a colorimetric parameter for classifying illuminants and real lamps, taking into account both the volume (number of distinguishable colors) and the shape of the associated color solid.

Consequently, although the original aim of this work was the improvement of the method for calculating and plotting optimal colors, originally developed by MacAdam, the analysis of the shown findings have given rise to very interesting preliminary conclusions about the colorimetry of light sources and color perception, which are worthwhile to study in the coming years. The potential applications of this work, and those derived from it, could be numerous for lighting design, color imaging, color perception in animal vision, etc.

\section{ACKNOWLEDGMENTS}

This research was supported by the Ministerio de Educación y Ciencia, Spain, under grant DPI2005-08999-C0202, and by the Conselleria d'Empresa, Universitat i Ciència of the Generalitat Valenciana, Spain, under grant IIARC0/2004/59. The authors thank the reviewers for their advice, helpful comments, and suggestions, particularly for the comments incorporated in our discussion.

Corresponding author F. Martínez-Verdú can be reached by e-mail at verdu@ua.es.

\section{REFERENCES}

1. R. G. Kuehni, Color Space and Its Divisions: Color Order from Antiquity to the Present (Wiley, 2003).

2. G. Wyszecki and W. S. Stiles, Color Science: Concepts and Methods, Quantitative Data and Formulae, 2nd ed. (Wiley, 1982), pp. 179-184.

3. R. S. Berns, Billmeyer and Saltzman's Principles of Color Technology, 3rd ed. (Wiley, 2000).

4. D. L. MacAdam, "Maximum visual efficiency of colored materials," J. Opt. Soc. Am. 25, 316-367 (1935).

5. D. L. MacAdam, "Theory of the maximum visual efficiency of colored materials," J. Opt. Soc. Am. 25, 249-252 (1935).

6. E. Schrödinger, "Theorie der Pigmente von grösster Leuchtkraft," Ann. Phys. 62, 603-622 (1920).

7. S. Rösch, "Fortschritte der Mineral," Kristallogr. Petrogr. 13, 143 (1929).

8. R. G. Kuehni, Color Space and Its Divisions: Color Order from Antiquity to the Present (Wiley, 2003), pp. 91, 359.

9. R. S. Berns, Billmeyer and Saltzman's Principles of Color Technology, 3rd ed. (Wiley, 2000), pp. 62, 143.

10. R. W. G. Hunt, The Reproduction of Colour, 6th ed. (Wiley, 2004), pp. 88-90.

11. T. Seim and A. Valberg, "Towards a uniform color space: a better formula to describe the Munsell and OSA color scales," Color Res. Appl. 11, 11-24 (1986).

12. G. Cui, M. R. Luo, B. Rigg, G. Roesler, and K. Witt, "Uniform colour spaces based on the DIN99 colourdifference formula," Color Res. Appl. 27, 282-290 (2002).

13. CIE, A Colour Appearance Model for Colour Management Systems: CIECAM02, CIE 159:2004 (Commission Internationale de l'Eclairage, 2004).

14. M. R. Pointer, "The gamut of real surface colours," Color Res. Appl. 5, 145-155 (1980).

15. M. R. Pointer, "Request for real surface colours," Color Res. Appl. 27, 374 (2002).

16. N. Ohta and A. Robertson, Colorimetry: Fundamentals and Applications (Wiley, 2005), pp. 215.

17. G. Wyszecki and W. S. Stiles, Color Science: Concepts and Methods, Quantitative Data and Formulae, 2nd ed. (Wiley, 1982), pp. 725-735.

18. CIE, Recommended Practice for Tabulating Spectral Data 
for Use in Colour Computations, CIE 167:2005 (Commission Internationale de l'Eclairage, 2005).

19. CIE, Colorimetry, 3rd ed., CIE 15:2004 (Commission Internationale de l'Eclairage, 2004).

20. CIE, Method of Measuring and Specifying Colour Rendering Properties of Light Sources, CIE 13.3:1995 (Commission Internationale de l'Eclairage, 1995).

21. CIE, CIE Collection 1999, Vision and Colour, Physical Measurement of Light and Radiation, Research Note: Colour Rendering, TC 1-33 Closing Remarks, CIE 135/ 2:1999 (Commission Internationale de l'Eclairage, 1999).

22. C. van Trigt, "Color rendering, a reassessment," Color Res. Appl. 24, 197-206 (1999).

23. J. A. Worthey, "Color rendering: asking the question," Color Res. Appl. 28, 403-412 (2003).

24. D. B. Judd and G. Wyszecki, Color in Business, Science, and Industry, 3rd ed. (Wiley, 1975), pp. 267.

25. R. W. G. Hunt, The Reproduction of Colour, 6th ed. (Wiley, 2004), pp. 162-163.

26. R. G. Kuehni, Color Space and Its Divisions: Color Order from Antiquity to the Present (Wiley, 2003), pp. 202.

27. S. D. Hordley and G. D. Finlayson, "Reevaluation of color constancy algorithm performance," J. Opt. Soc. Am. A 23, 1008-1020 (2006).

28. A. Lewis and L. Zhaoping, "Are cone sensitivities determined by natural color statistics?" J. Vision 6, 285-302 (2006).

29. Y. Nayatani, "Development of chromatic adaptation transforms and concept for their classification," Color Res. Appl. 31, 205-217 (2006).

30. S. D. Hordley, "Scene illuminant estimation: past, present, and future," Color Res. Appl. 31, 303-314 (2006).

31. D. H. Brainard, P. Longère, P. B. Delahunt, W. T. Freeman, J. M. Kraft, and V. Xiao, "Bayesian model of human color constancy," J. Vision 6, 1267-1281 (2006).

32. E. Perales, F. Martínez-Verdú, V. Viqueira, M. J. Luque, and P. Capilla, "Computing the number of distinguishable colors under several illuminants and light sources," in Proceedings of Third IS\&T European Conference on Colour Graphics, Imaging and Vision (Society for Imaging Science and Technology, 2006), pp. 414-419.

33. J. Krauskopf, "Higher order color mechanisms," in Color Vision: From Genes to Perception, K. R. Gegenfurtner and L. T. Sharpe, eds. (Cambridge U. Press, 1999), pp. 310.

34. U. Steingrímsson, K. Simon, W. Steiger, and K. Schläpfer, "The gamut obtainable with surface colors," in Proceedings of First IS\&T European Conference on Colour Graphics, Imaging and Vision (Society for Imaging Science and Technology, 2002), pp. 287-291.

35. CIE, Criteria for the Evaluation of Extended-Gamut Colour Encodings, CIE 168:2005 (Commission Internationale de l'Eclairage, 2005).

36. F. Martínez-Verdú, M. J. Luque, P. Capilla, and J. Pujol, "Concerning the calculation of the color gamut in a digital camera," Color Res. Appl. 31, 399-410 (2006). 